\title{
Minimal generating sets of Reidemeister moves
}

\author{
Michael Polyak*
}

\begin{abstract}
It is well known that any two diagrams representing the same oriented link are related by a finite sequence of Reidemeister moves $\Omega 1, \Omega 2$ and $\Omega 3$. Depending on orientations of fragments involved in the moves, one may distinguish 4 different versions of each of the $\Omega 1$ and $\Omega 2$ moves, and 8 versions of the $\Omega 3$ move. We introduce a minimal generating set of 4 oriented Reidemeister moves, which includes two $\Omega 1$ moves, one $\Omega 2$ move, and one $\Omega 3$ move. We then study which other sets of up to 5 oriented moves generate all moves, and show that only few of them do. Some commonly considered sets are shown not to be generating. An unexpected non-equivalence of different $\Omega 3$ moves is discussed.
\end{abstract}

Mathematics Subject Classification (2010). 57M25, 57M27.

Keywords. Reidemeister moves, knot and link diagrams.

\section{Introduction}

A standard way to describe a knot or a link in $\mathbb{R}^{3}$ is via its diagram, i.e., a generic plane projection of the link such that the only singularities are transversal double points, endowed with the over/undercrossing information at each double point. Two diagrams are equivalent if there is an orientation-preserving diffeomorphism of the plane that carries one diagram to the other diagram. A classical result of Reidemeister [6] states that any two diagrams of isotopic links are related by a finite sequence of simple moves ${ }^{1} \Omega 1, \Omega 2$, and $\Omega 3$, shown in Figure 1 .

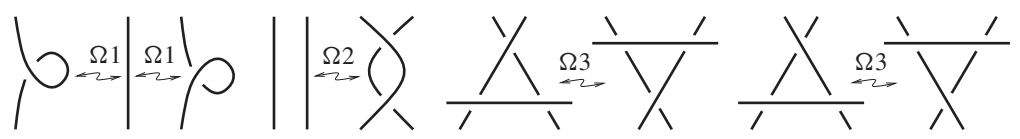

Figure 1. Reidemeister moves.

\footnotetext{
*We are grateful to O. Viro for posing the problem and to S. Chmutov for valuable discussions. The author was supported by an ISF grant 1261/05 and by the J. Steiner foundation.

${ }^{1}$ Our notation makes no distinction between a move and the inverse move.
} 
Here we assume that two diagrams $D, D^{\prime}$ related by a move coincide outside an oriented embedded disk $C \subset \mathbb{R}^{2}$ (with an orientation of $C$ induced by the standard orientation of $\mathbb{R}^{2}$ ), called the changing disk, and look as a corresponding pair $R$, $R^{\prime}$ of arc diagrams in Figure 1 inside $C$. In other words, there are two orientationpreserving diffeomorphisms $f, f^{\prime}: C \rightarrow B^{2}$ of $C$ to the standard oriented 2-disk $B^{2}$, such that $f_{\partial C}=f_{\partial C}^{\prime}$ and $f(C \cap D)=R, f^{\prime}\left(C \cap D^{\prime}\right)=R^{\prime}$.

To deal with oriented links we consider oriented diagrams. Depending on orientations of fragments involved in the moves, one may distinguish four different versions of each of the $\Omega 1$ and $\Omega 2$ moves, and eight versions of the $\Omega 3$ move; see Figures 2, 3 , and 4 , respectively.
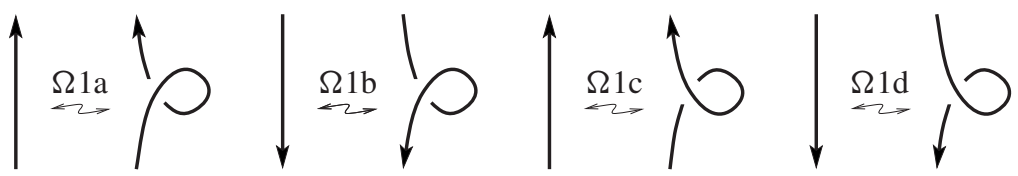

Figure 2. Oriented Reidemeister moves of type 1.
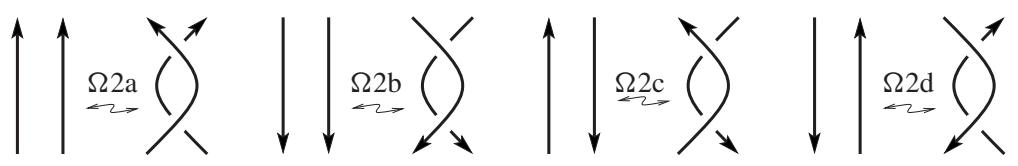

Figure 3. Oriented Reidemeister moves of type 2.

When one checks that a certain function of knot or link diagrams defines a link invariant, it is important to minimize the number of moves. We will call a collection $S$ of oriented Reidemeister moves a generating set if any oriented Reidemeister move $\Omega$ may be obtained by a finite sequence of isotopies and moves from the set $S$ inside the changing disk of $\Omega$.

While some dependencies between oriented Reidemeister moves are well known, the standard generating sets of moves usually include six different $\Omega 3$ moves, see e.g. Kauffman [3]. For sets with a smaller number of $\Omega 3$ moves there seems to be a number of different, often contradictory, results. In particular, Turaev [7], proof of Theorem 5.4, introduces a set of five oriented Reidemeister moves with only one $\Omega 3$ move. There is no proof (and in fact we will see in Section 3 that this particular set is not generating), with the only comment being a reference to a figure where, unfortunately, a move $\Omega 2$ which does not belong to the set is used. Wu [9] uses the same set of moves citing [7] but additionally incorrectly puts the total number of oriented $\Omega 3$ moves at 12 (instead of 8). Kauffman [3], p. 90, includes as an exercise a set of all $\Omega 1$ and $\Omega 2$ moves together with two $\Omega 3$ moves. Meyer [4] uses a set with four $\Omega 1$, two $\Omega 2$, and two $\Omega 3$ moves and states (again without a proof) that the minimal number of needed $\Omega 3$ moves is two. The number of $\Omega 3$ moves used by 

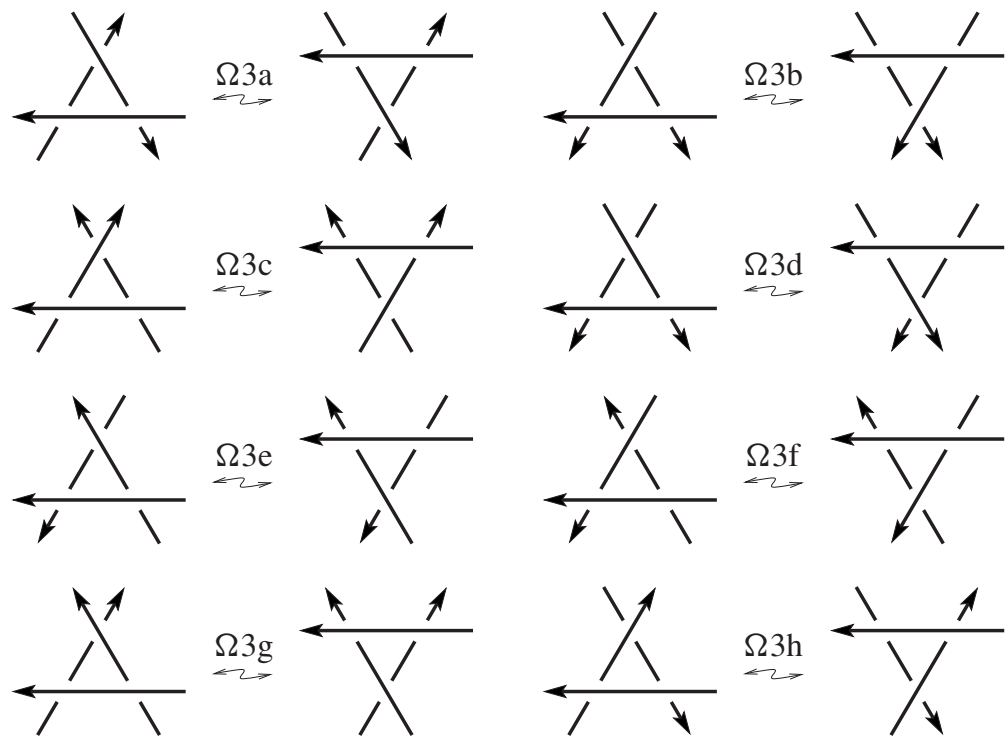

Figure 4. Oriented Reidemeister moves of type 3.

Östlund [5] is also two, but his classification of $\Omega 3$ moves works only for knots and is non-local (depending on the cyclic order of the fragments along the knot). Series of exercises in Chmutov et al. [1] (unfortunately without proofs) suggest that only one $\Omega 3$ suffices, but this involves all $\Omega 2$ moves. These discrepancies are most probably caused by the fact that while many people needed some statement of this kind, it was only an auxiliary technical statement, a proof of which would be too long and would take the reader away from the main subject. So only a brief comment was usually made. We believe that it is time for a careful treatment. In this note we introduce a simple generating set of four Reidemeister moves, which includes two $\Omega 1$ moves, one $\Omega 2$ move and one $\Omega 3$ move.

Theorem 1.1. Let $D$ and $D^{\prime}$ be two diagrams in $\mathbb{R}^{2}$ representing the same oriented link. Then one may pass from $D$ to $D^{\prime}$ by isotopy and a finite sequence offour oriented Reidemeister moves $\Omega 1 \mathrm{a}, \Omega 1 \mathrm{~b}, \Omega 2 \mathrm{a}$, and $\Omega 3 \mathrm{a}$, shown in Figure 5.
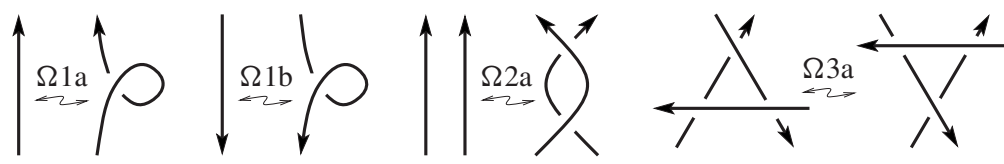

Figure 5. A generating set of Reidemeister moves.

This generating set of Reidemeister moves has the minimal number of generators. 
Indeed, it is easy to show that any generating set should contain at least one move of each of the types two and three; Lemma 2.2 in Section 3 implies that there should be at least two moves of type one. Thus any generating set of Reidemeister moves should contain at least four moves.

Our choice of the move $\Omega 3 \mathrm{a}$ as a generator may look unusual since this move (called a cyclic $\Omega 3$ move, see e.g. [3]) is rarely included in the list of generators, contrary to a more common move $\Omega 3 \mathrm{~b}$ which is the standard choice motivated by braid theory. ${ }^{2}$ The reason is that, unexpectedly, these moves have different properties, as we discuss in detail in Section 3. Indeed, Theorem 1.2 below implies that any generating set of Reidemeister moves which includes $\Omega 3 \mathrm{~b}$ has at least five moves. If we consider sets of five Reidemeister moves which contain $\Omega 3 \mathrm{~b}$, then it turns out that out of all combinations of $\Omega 1$ and $\Omega 2$ moves only 4 sets generate all Reidemeister moves. The only freedom is in the choice of $\Omega 1$ moves, while $\Omega 2$ moves are uniquely determined:

Theorem 1.2. Let $S$ be a set of at most five Reidemeister moves which contains only one move, $\Omega 3 \mathrm{~b}$, of type three. The set $S$ generates all Reidemeister moves if and only if $S$ contains $\Omega 2 \mathrm{c}$ and $\Omega 2 \mathrm{~d}$, and one of the pairs ( $\Omega 1 \mathrm{a}, \Omega 1 \mathrm{~b}),(\Omega 1 \mathrm{a}, \Omega 1 \mathrm{c})$, $(\Omega 1 \mathrm{~b}, \Omega 1 \mathrm{~d})$, or $(\Omega 1 \mathrm{c}, \Omega 1 \mathrm{~d})$.

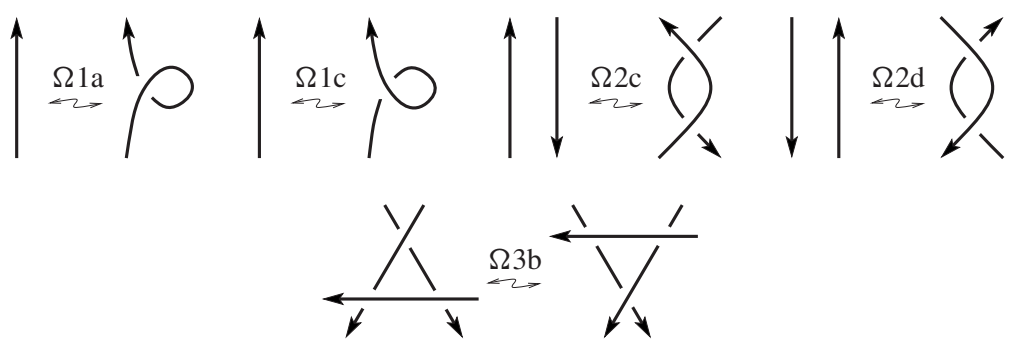

Figure 6. A generating set of Reidemeister moves containing $\Omega 3 \mathrm{~b}$.

One of these generating sets is shown in Figure 6. It is interesting to note that while (by Markov's theorem) the set $\Omega 1 \mathrm{a}, \Omega 1 \mathrm{c}, \Omega 2 \mathrm{a}, \Omega 2 \mathrm{~b}$ and $\Omega 3 \mathrm{~b}$ shown in Figure 7 allows one to pass between any two braids with isotopic closures, this set is not sufficient to connect any pair of general diagrams representing the same link. This means that some extra moves should appear in the process of transforming a general link diagram into a closed braid. And indeed, in all known algorithms of such a transformation the additions moves occur. For example, in Vogel's algorithm [8] the moves $\Omega 2 \mathrm{c}$ and $\Omega 2 \mathrm{~d}$ are the main steps of the algorithm.

Even more unexpected is the fact that all type one moves together with $\Omega 2 \mathrm{a}, \Omega 2 \mathrm{c}$ (or $\Omega 2 \mathrm{~d}$ ) and $\Omega 3 \mathrm{~b}$ are also insufficient; see Figure 8 .

\footnotetext{
${ }^{2}$ This is the only $\Omega 3$ move with all three positive crossings.
} 
Minimal generating sets of Reidemeister moves
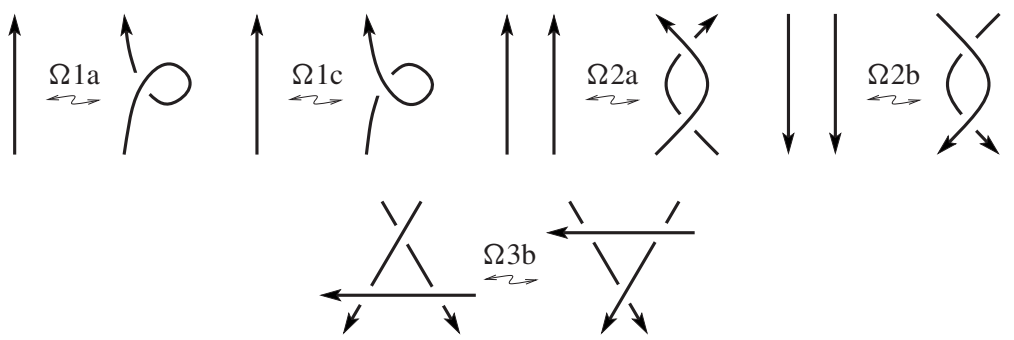

Figure 7. This is not a generating set.
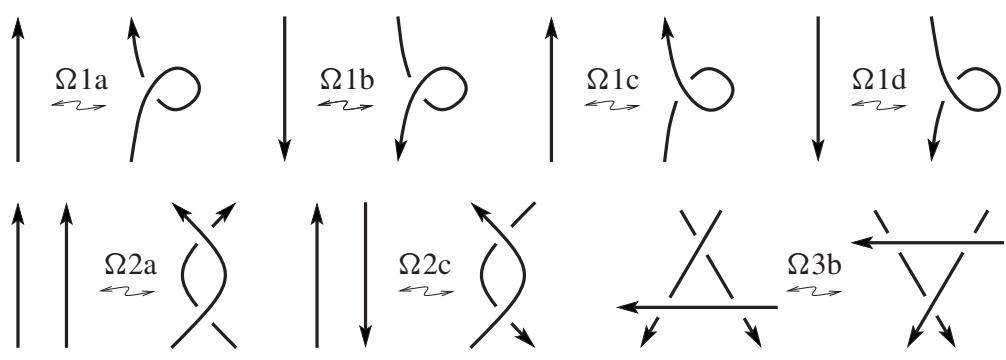

Figure 8. Another set which is not generating.

Remark 1.3. For non-oriented links a sequence of Reidemeister moves can be arranged in such a form that first a number of $\Omega 1$ moves are performed, then $\Omega 2$ moves are performed, after this $\Omega 3$ moves are performed, and finally $\Omega 2$ moves have to be performed again; see [2]. It would be interesting to find such a theorem for oriented case.

All our considerations are local, and no global realization restrictions are involved. Therefore all our results hold also for virtual links.

Section 2 is dedicated to the proof of Theorem 1.1. In Section 3 we discuss various generating sets which contain $\Omega 3 \mathrm{~b}$ and prove Theorem 1.2

\section{A minimal set of oriented Reidemeister moves}

In this section we prove Theorem 1.1 in several easy steps. The first step is to obtain $\Omega 2 \mathrm{c}, \Omega 2 \mathrm{~d}$ :

Lemma 2.1. The move $\Omega 2 \mathrm{c}$ may be realized by a sequence of $\Omega 1 \mathrm{a}, \Omega 2 \mathrm{a}$ and $\Omega 3 \mathrm{a}$ moves. The move $\Omega 2 \mathrm{~d}$ may be realized by a sequence of $\Omega 1 \mathrm{~b}, \Omega 2 \mathrm{a}$ and $\Omega 3 \mathrm{a}$ moves. 
Proof.

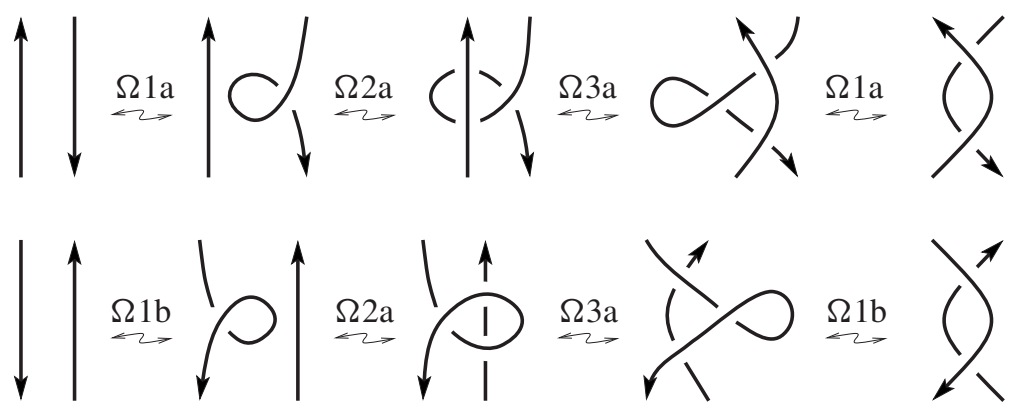

Now the remaining moves of type one may be obtained as in [5]:

Lemma 2.2 ([5]). The move $\Omega 1 \mathrm{c}$ may be realized by a sequence of $\Omega 1 \mathrm{~b}$ and $\Omega 2 \mathrm{~d}$ moves. The move $\Omega 1 \mathrm{~d}$ may be realized by a sequence of $\Omega 1 \mathrm{a}$ and $\Omega 2 \mathrm{c}$ moves.

Proof.
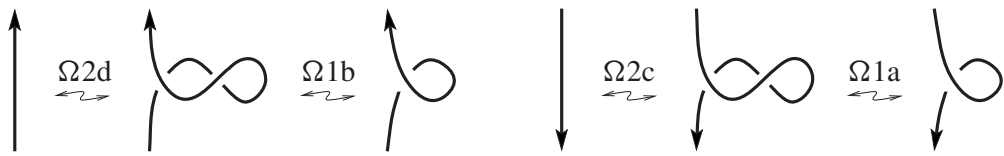

This concludes the treatment of all $\Omega 1$ and $\Omega 2$ moves, except for $\Omega 2 b$; we will take care of it later. Having in mind Section 3, where we will deal with $\Omega 3$ b instead of $\Omega 3 \mathrm{a}$, we will first consider $\Omega 3 \mathrm{~b}$ :

Lemma 2.3. The move $\Omega 3 \mathrm{~b}$ may be realized by a sequence of $\Omega 2 \mathrm{c}, \Omega 2 \mathrm{~d}$, and $\Omega 3 \mathrm{a}$ moves.

Proof.
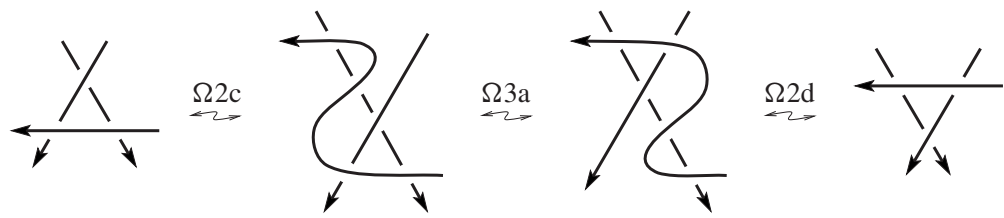

To deal with $\Omega 2 \mathrm{~b}$ we will need another move of type three: 
Lemma 2.4. The move $\Omega 3 \mathrm{c}$ may be realized by a sequence of $\Omega 2 \mathrm{c}, \Omega 2 \mathrm{~d}$, and $\Omega 3 \mathrm{a}$ moves.

Proof.
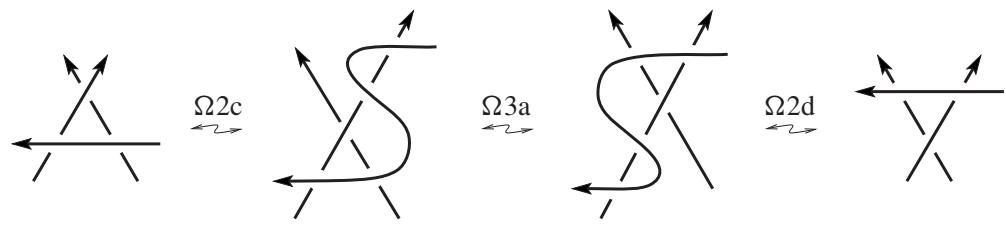

At this stage we can obtain the remaining move $\Omega 2 \mathrm{~b}$ of type two:

Lemma 2.5. The move $\Omega 2 \mathrm{~b}$ may be realized by a sequence of $\Omega 1 \mathrm{~d}, \Omega 2 \mathrm{c}$ and $\Omega 3 \mathrm{c}$ moves.

Proof.

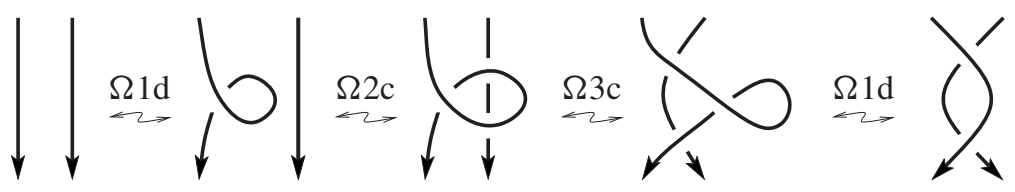

To conclude the proof of Theorem 1.1, it remains to obtain $\Omega 3 d-\Omega 3$ h. Since by now we have in our disposal all moves of type two, this becomes an easy exercise:

Lemma 2.6. The moves $\Omega 3 \mathrm{~d}-\Omega 3 \mathrm{~h}$ of type three may be realized by a sequence of type two moves, $\Omega 3 \mathrm{a}$, and $\Omega 3 \mathrm{~b}$.

Proof. We realize moves $\Omega 3 \mathrm{~d}-\Omega 3 \mathrm{~h}$ as shown in rows $1-5$ of the figure below, using $\Omega 3 \mathrm{f}$ to get $\Omega 3 \mathrm{~g}$, and $\Omega 3 \mathrm{~g}$ to get $\Omega 3 \mathrm{~h}$ :
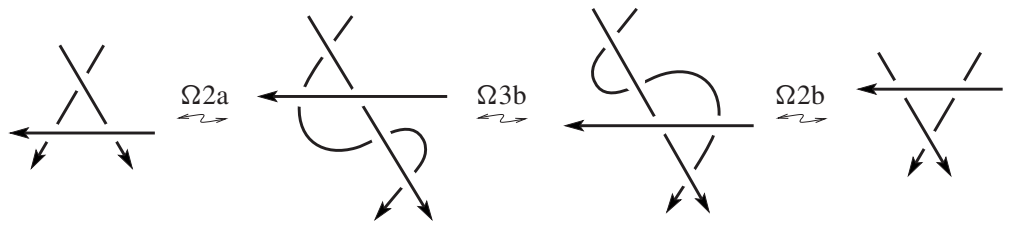

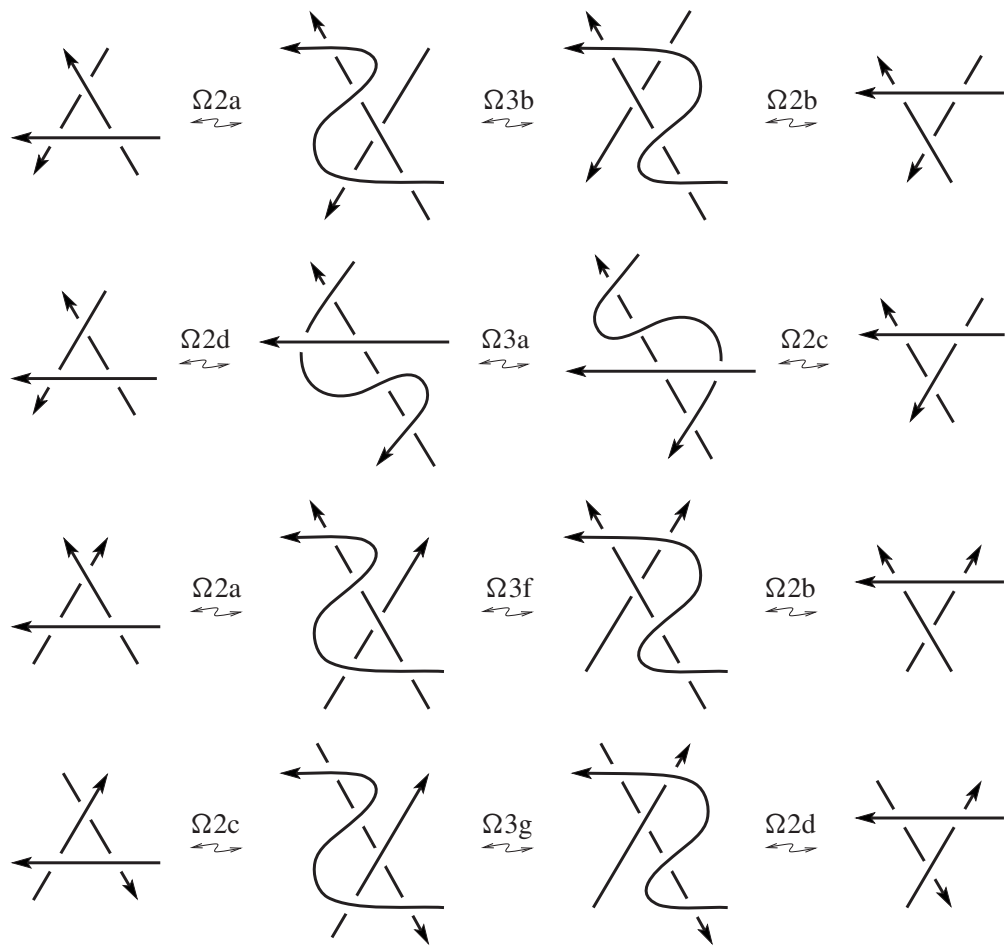

Remark 2.7. There are other generating sets which include $\Omega 3 \mathrm{a}$. In particular, $\Omega 1 \mathrm{a}$, $\Omega 1 \mathrm{~b}, \Omega 2 \mathrm{~b}$ and $\Omega 3 \mathrm{a}$ also give a generating set. To adapt the proof of Theorem 1.1 to this case, one needs only a slight modification of Lemma 2.1. All other lemmas do not change.

\section{Other sets of Reidemeister moves}

In this section we discuss other generating sets and prove Theorem 1.2. Unexpectedly, different $\Omega 3$ moves have different properties as far as generating sets of Reidemeister moves are concerned. Let us study the case of $\Omega 3 \mathrm{~b}$ in more detail, due to its importance for braid theory.

In a striking contrast to Theorem 1.1 which involves $\Omega 3$ a, Theorem 1.2 implies that there does not exist a generating set of four moves which includes $\Omega 3 \mathrm{~b}$. It is natural to ask where does the proof in Section 2 breaks down if we attempt to replace $\Omega 3$ a with $\Omega 3 b$.

The only difference between $\Omega 3 \mathrm{a}$ and $\Omega 3 \mathrm{~b}$ may be pinpointed to Lemma 2.1: it does not have an analogue with $\Omega 3 \mathrm{~b}$ replacing $\Omega 3 \mathrm{a}$, as we will see in the proof of Lemma 3.8 below. 
An analogue of Lemma 2.3 is readily shown to exist. Indeed, $\Omega 3$ a may be realized by a sequence of $\Omega 2 \mathrm{c}, \Omega 2 \mathrm{~d}$ and $\Omega 3 \mathrm{~b}$ moves, as illustrated below:
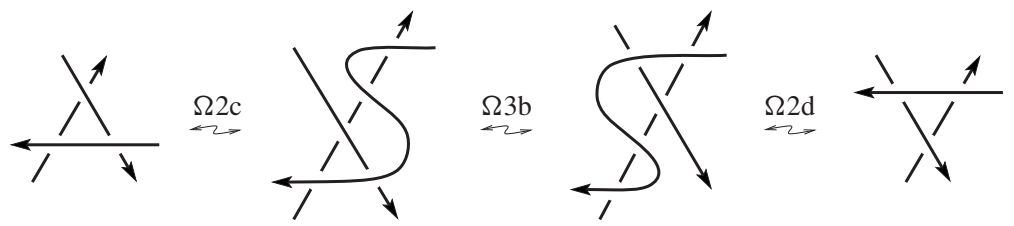

Using this fact instead of Lemma 2.3, together with the rest of Lemmas 2.2-2.6, implies that $\Omega 1 \mathrm{a}$ and $\Omega 1 \mathrm{~b}$, taken together with $\Omega 2 \mathrm{c}, \Omega 2 \mathrm{~d}$, and $\Omega 3 \mathrm{~b}$, indeed provide a generating set. Moreover, a slight modification of Lemma 2.2 shows that any of the other three pairs of $\Omega 1$ moves in the statement of Theorem 1.2 may be used instead of $\Omega 1 \mathrm{a}$ and $\Omega 1 \mathrm{~b}$. Thus we see that all sets described in Theorem 1.2 are indeed generating and obtain the "if" part of the theorem. It remains to prove the "only if" part of Theorem 1.2, i.e., to show that other combinations of four $\Omega 1$ and $\Omega 2$ moves, taken together with $\Omega 3 \mathrm{~b}$, do not result in generating sets. We will proceed in three steps:

Step 1: Prove that any such generating set should contain at least two $\Omega 1$ moves and to eliminate two remaining pairs $(\Omega 1 \mathrm{a}, \Omega 1 \mathrm{~d})$ and $(\Omega 1 \mathrm{~b}, \Omega 1 \mathrm{c})$ of $\Omega 1$ moves.

Step 2: Prove that any such generating set should contain at least two $\Omega 2$ moves and to eliminate pairs $(\Omega 2 \mathrm{a}, \Omega 2 \mathrm{c}),(\Omega 2 \mathrm{a}, \Omega 2 \mathrm{~d}),(\Omega 2 \mathrm{~b}, \Omega 2 \mathrm{c})$, and $(\Omega 2 \mathrm{~b}, \Omega 2 \mathrm{~d})$.

Step 3: Eliminate the remaining pair $(\Omega 2 \mathrm{a}, \Omega 2 \mathrm{~b})$.

The remainder of this section is dedicated to these three steps. Step 1 is the simplest and is given by Lemma 3 below. Step 2 is the most complicated; it is given by Corollaries 3.5 and 3.6. Step 3 is relatively simple and is given by Lemma 3.8.

To show that a certain set of Reidemeister moves is not generating, we will construct an invariant of these moves which, however, is not preserved under the set of all Reidemeister moves. The simplest classical invariants of this type are the writhe $w$ and the winding number rot of the diagram. The winding number of the diagram grows (respectively drops) by one under $\Omega 1$ b and $\Omega 1$ d (respectively $\Omega 1$ a and $\Omega 1 \mathrm{c}$ ). The writhe of the diagram grows (respectively drops) by one under $\Omega 1 \mathrm{a}$ and $\Omega 1 \mathrm{~b}$ (respectively $\Omega 1 \mathrm{c}$ and $\Omega 1 \mathrm{~d}$ ). Moves $\Omega 2$ and $\Omega 3$ do not change $w$ and rot. These simple invariants suffice to deal with moves of type one (see e.g. [5]):

Lemma 3.1 ([5]). Any generating set of Reidemeister moves contains at least two $\Omega 1$ moves. None of the two pairs $(\Omega 1 \mathrm{a}, \Omega 1 \mathrm{~d})$ or $(\Omega 1 \mathrm{~b}, \Omega 1 \mathrm{c})$, taken together with all $\Omega 2$ and $\Omega 3$ moves, gives a generating set.

Proof. Indeed, both $\Omega 1$ a and $\Omega 1$ d preserve $w+$ rot, so this pair (or any of them separately) together with $\Omega 2$ and $\Omega 3$ moves cannot generate all Reidemeister moves. The case of $\Omega 1 \mathrm{~b}$ and $\Omega 1 \mathrm{c}$ is obtained by the reversal of an orientation (of all components) of the link. 
This concludes step 1 of the proof. Let us proceed with step 2. Here the situation is quite delicate since the standard algebraic/topological invariants, reasonably well behaved under compositions, cannot be applied. The reason can be explained by a simple example: suppose that we want to show that $\Omega 2 \mathrm{~d}$ cannot be obtained by a sequence of Reidemeister moves which includes $\Omega 2 \mathrm{c}$. Then our invariant should be preserved under $\Omega 2 \mathrm{c}$ and distinguish two tangles shown in Figure 9a. However, if we compose them with a crossing, as shown in Figure 9b, we may pass from one to another by $\Omega 2 \mathrm{c}$. Thus the invariant should not survive composition of tangles.

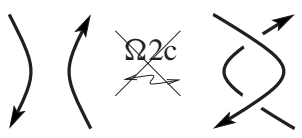

a

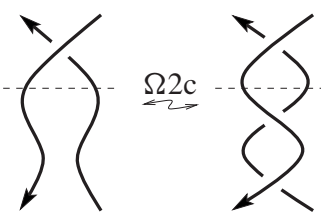

b

Figure 9. Composition destroys inequivalence.

Instead we will use a certain notion of positivity, which is indeed destroyed by such compositions. It is defined as follows. Let $D$ be a $(2,2)$-tangle diagram with two oriented ordered components $D_{1}, D_{2}$. Decorate all arcs of both components of $D$ with an integer weight by the following rule. Start walking on $D_{1}$ along the orientation. Assign zero to the initial arc. Each time when we pass an overcrossing (we do not count undercrossings) with $D_{2}$, we add a sign (the local writhe) of this overcrossing to the weight of the previous arc. Now start walking on $\mathrm{D}_{2}$ along the orientation. Again, assign zero to the initial arc. Each time when we pass an undercrossing (now we do not count overcrossings) with $D_{1}$, we add a sign of this undercrossing to the weight of the previous arc. See Figure 10a. Two simple examples are shown in Figure 10b, c.
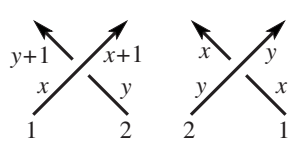

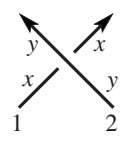

a
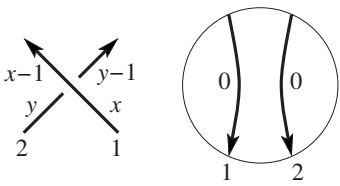

b

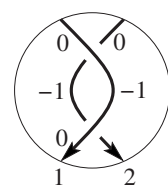

$\mathrm{c}$

Figure 10. Weights of diagrams.

We call a component positively weighted if weights of all its arcs are non-negative. For instance, both components of the (trivial) tangle in Figure 10b are positively weighted. None of the components of a diagram in Figure 10c are positively weighted (since the weights of the middle arcs on both components are -1 ). The behavior of positivity under Reidemeister moves is considered in the next lemmas.

Denote by $S_{\mathrm{b}}$ the set which consists of all $\Omega 1$ moves and $\Omega 3 \mathrm{~b}$. 
Lemma 3.2. Let $D$ be a $(2,2)$-tangle diagram with both positively weighted components. Then both components of a diagram obtained from it by a sequence of moves which belong to $S_{\mathrm{b}} \cup \Omega 2 \mathrm{a}$ are also positively weighted.

Proof. Indeed, an application of a first Reidemeister move does not change this property since we count only intersections of two different components. An application of $\Omega 2 \mathrm{a}$ adds (or removes) two crossings on each component in such a way that walking along a component we first meet a positive crossing and then the negative one. So the weights of the middle arcs are either the same or larger than on the surrounding arcs; see Figure 11a. An application of $\Omega 3 \mathrm{~b}$ preserves the weights since $\Omega 3 \mathrm{~b}$ involves only positive crossings.

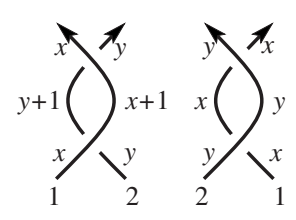

a

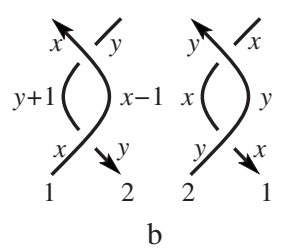

Figure 11. Weights and Reidemeister moves of type two.

Lemma 3.3. Let $D$ be a $(2,2)$-tangle diagram with a positively weighted second component. Then any diagram obtained from it by $\Omega 2 \mathrm{c}$ also has a positively weighted second component.

Proof. An application of $\Omega 2 \mathrm{c}$ may add (or remove) two undercrossings on $D_{2}$, but in such a way that we first meet a positive undercrossing and then the negative one, so the weight of a middle arc is larger than on the surrounding arcs, see Figure $11 \mathrm{~b}$.

Lemma 3.4. Let $D$ be a $(2,2)$-tangle diagram with a positively weighted first component. Then any diagram obtained from it by $\Omega 2 \mathrm{~d}$ also has a positively weighted first component.

Proof. An application of $\Omega 2 \mathrm{~d}$ may add (or remove) two overcrossings on $D_{1}$, but in such a way that we first meet a positive overcrossing and then the negative one. So the weight of a middle arc is larger than on the surrounding arcs; see Figure 11c.

Comparing Figures $10 \mathrm{~b}$ and $10 \mathrm{c}$ we conclude

Corollary 3.5. None of the two sets $S_{\mathrm{b}} \cup \Omega 2 \mathrm{a} \cup \Omega 2 \mathrm{c}$ and $S_{\mathrm{b}} \cup \Omega 2 \mathrm{a} \cup \Omega 2 \mathrm{~d}$ generates $\Omega 2 b$. 
The reversal of orientations (of both components) of the tangle in the above construction gives

Corollary 3.6. None of the two sets $S_{\mathrm{b}} \cup \Omega 2 \mathrm{~b} \cup \Omega 2 \mathrm{c}$ and $S_{\mathrm{b}} \cup \Omega 2 \mathrm{~b} \cup \Omega 2 \mathrm{~d}$ generates $\Omega 2 \mathrm{a}$.

Remark 3.7. In [7], Theorem 5.4 (and later [9]) the set $S_{\mathrm{b}} \cup \Omega 2 \mathrm{a} \cup \Omega 2 \mathrm{c}$ is considered as a generating set. Fortunately (V. Turaev, personal communication), an addition of $\Omega 2 \mathrm{~d}$ does not change the proof of the invariance in [7], Theorem 5.4.

Note that the above corollaries imply that any generating set $S$ which contains only one move, $\Omega 3 \mathrm{~b}$, of type three, should contain at least two $\Omega 2$ moves. This concludes step 2 of the proof.

Since at the same time such a set $S$ should contain at least two $\Omega 1$ moves by Lemma, we conclude that if $S$ consists of five moves, there should be exactly two $\Omega 2$ moves and two $\Omega 1$ moves. This simple observation allows us to eliminate the last remaining case:

Lemma 3.8. Let $S$ be a set which consists of two $\Omega 1$ moves, $\Omega 2 \mathrm{a}, \Omega 2 \mathrm{~b}$, and $\Omega 3 \mathrm{~b}$. Then $S$ is not generating.

Proof. Given a link diagram, smooth all double points of the diagram respecting the orientation, as illustrated in Figure 12.
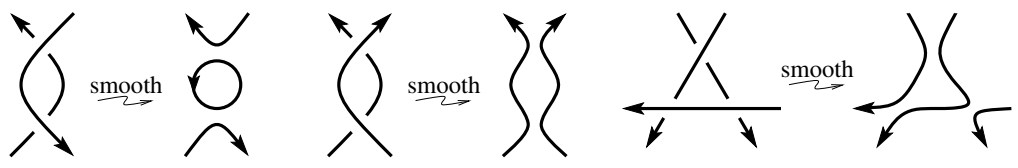

Figure 12. Smoothing the diagram respecting the orientation.

Count the numbers $C^{-}$and $C^{+}$of clockwise and counter-clockwise oriented circles of the smoothed diagram, respectively. Note that $\Omega 2 \mathrm{a}, \Omega 2 \mathrm{~b}$, and $\Omega 3 \mathrm{~b}$ preserve an isotopy class of the smoothed diagram, thus preserve both $C^{+}$and $C^{-}$. On the other hand, $\Omega 1 \mathrm{~b}$ and $\Omega 1 \mathrm{~d}$ add one to $C^{+}$, and $\Omega 1 \mathrm{a}, \Omega 1 \mathrm{c}$ add one to $C^{-}$. Thus if $S$ contains $\Omega 1 \mathrm{a}$ and $\Omega 1 \mathrm{c}$, all moves of $S$ preserve $C^{+}$. The case of $\Omega 1 \mathrm{~b}$ and $\Omega 1 \mathrm{~d}$ is obtained by the reversal of an orientation (of all components) of the link. If $S$ contains $\Omega 1 \mathrm{a}$ and $\Omega 1 \mathrm{~b}$, all moves of $S$ preserve $C^{+}+C^{-}-w$. Similarly, if $S$ contains $\Omega 1 \mathrm{c}$ and $\Omega 1$ d, all moves of $S$ preserve $C^{+}+C^{-}+w$. In all the above cases, moves from $S$ can not generate $\Omega 2 \mathrm{c}, \Omega 2 \mathrm{~d}$ since each of $\Omega 2 \mathrm{c}$ and $\Omega 2 \mathrm{~d}$ may change $C^{+}$as well as $C^{+}+C^{-} \pm w$ (while preserving $w$ and $C^{+}-C^{-}=\operatorname{rot}$ ).

This concludes the proof of Theorem 1.2. 


\section{References}

[1] S. Chmutov, S. Duzhin, and J. Mostovoy, Introduction to Vassiliev knot invariants. Preliminary draft version of a book about Chord Diagrams.

http://www.math.ohio-state.edu/ chmutov/preprints/\#

[2] A. Coward, Ordering the Reidemeister moves of a classical knot. Algebr. Geom. Topol. 6 (2006), 659-671. MR 2240911 Zbl 1095.57005

[3] L. H. Kauffman, Knots and physics. 3rd ed., Ser. Knots Everything 1, 3rd ed., World Scientific, Singapore 2001. MR 1858113 Zbl 1057.57001

[4] D. A. Meyer, State models for link invariants from the classical Lie groups. In Knots 90 (Osaka, 1990), Walter de Gruyter, Berlin 1992, 559-592. MR 1177447 Zbl 0770.57004

[5] O.-P. Östlund, Invariants of knot diagrams and relations among Reidemeister moves. $J$. Knot Theory Ramifications 10 (2001), 1215-1227. MR 1871226 Zbl 0998.57021

[6] K. Reidemeister, Knoten und Gruppen. Abh. Math. Sem. Univ. Hamburg 5 (1926) , 7-23. JFM 52.0578.04

[7] V. G. Turaev, The Yang-Baxter equation and invariants of links. Invent. Math. 92 (1988), 527-553. MR 939474 Zbl 0648.57003

[8] P. Vogel, Representation of links by braids: a new algorithm. Comment. Math. Helv. 65 (1990), 104-113. MR 1036132 Zbl 0703.57004

[9] F.Y. Wu, Knot theory and statistical mechanics. Rev. Modern Phys. 64 (1992), 1099-1131. MR 1187941

Received January 8, 2010

M. Polyak, Department of Mathematics, Technion - Israel Institute of Technology, Haifa 32000, Israel

E-mail: polyak@math.technion.ac.il 